\title{
Proficient brain for optimal performance: The MAP model perspective
}

Maurizio Bertollo, Selenia di Fronso, Edson Filho, Silvia Conforto, Maurizio Schmid, Laura Bortoli, Silvia Comani, Claudio Robazza

Background. The main goal of the present study was to explore theta and alpha Event Related Desynchronization/Synchronization (ERD/ERS) activity during shooting performance. We adopted the idiosyncratic framework of the multi-action plan (MAP) model to investigate different processing modes underpinning four types of performance. In particular, we were interested in examining the neural activity associated with optimal-automated (Type 1) and optimal-controlled (Type 2) performances.

Methods. Ten elite shooters ( 6 male and 4 female) with extensive international experience participated in the study. ERD/ERS analysis was used to investigate cortical dynamics during performance. A $4 \times 3$ (performance types $x$ time) repeated measures analysis of variance was performed to test the differences among the four types of performance during the three seconds preceding the shots for theta, low alpha, and high alpha frequency bands. The dependent variables were the ERD/ERS percentages in each frequency band (i.e., theta, low alpha, high alpha) for each electrode site across the scalp. This analysis was conducted on 120 shots for each participant in three different frequency bands and the individual data were then averaged.

Results. We found ERS to be mainly associated with optimal-automatic performance, in agreement with the "neural efficiency hypothesis". We also observed more ERD as related to optimal-controlled performance in conditions of "neural adaptability" and proficient use of cortical resources.

Discussion. These findings are congruent with the MAP conceptualization of four performance states, in which unique psychophysiological states underlie distinct performance-related experiences. From an applied point of view, our findings suggest that the MAP model can be used as a framework to develop performance enhancement strategies based on cognitive and neurofeedback techniques. 
1

2

3

4

5 'BIND - Behavioral Imaging and Neural Dynamics Center, University "G. d'Annunzio", Chieti-

6 Pescara, Chieti (Italy)

7 2Department of Medicine and Aging Sciences, University “G. d'Annunzio", Chieti-Pescara,

8 Chieti (Italy)

$9{ }^{3}$ School of Psychology, University of Central Lancashire, Preston, Lancashire (UK)

\section{Proficient brain for optimal performance: The MAP model perspective}

${ }^{4}$ Department of Engineering, Roma Tre University, Rome (Italy)

${ }^{5}$ Department of Neuroscience, Imaging and Clinical Sciences, University “G. d'Annunzio", Chieti-Pescara, Chieti (Italy)

\section{Corresponding author:} Maurizio Bertollo, PhD

BIND - Behavioral Imaging and Neural Dynamics Center Dept. of Medicine and Aging Sciences

University "G. d'Annunzio" of Chieti-Pescara

Via dei Vestini, 33

66013 Chieti - Italy

m.bertollo@unich.it

phone: +3908713554038 


\section{Abstract}

Background. The main goal of the present study was to explore theta and alpha Event Related Desynchronization/Synchronization (ERD/ERS) activity during shooting performance. We adopted the idiosyncratic framework of the multi-action plan (MAP) model to investigate different processing modes underpinning four types of performance. In particular, we were interested in examining the neural activity associated with optimal-automated (Type 1) and optimal-controlled (Type 2) performances.

Methods. Ten elite shooters (6 male and 4 female) with extensive international experience participated in the study. ERD/ERS analysis was used to investigate cortical dynamics during performance. A $4 \times 3$ (performance types $\times$ time) repeated measures analysis of variance was performed to test the differences among the four types of performance during the three seconds preceding the shots for theta, low alpha, and high alpha frequency bands. The dependent variables were the ERD/ERS percentages in each frequency band (i.e., theta, low alpha, high alpha) for each electrode site across the scalp. This analysis was conducted on 120 shots for each participant in three different frequency bands and the individual data were then averaged. Results. We found ERS to be mainly associated with optimal-automatic performance, in agreement with the "neural efficiency hypothesis". We also observed more ERD as related to optimal-controlled performance in conditions of "neural adaptability" and proficient use of cortical resources.

Discussion. These findings are congruent with the MAP conceptualization of four performance states, in which unique psychophysiological states underlie distinct performance-related experiences. From an applied point of view, our findings suggest that the MAP model can be used as a framework to develop performance enhancement strategies based on cognitive and neurofeedback techniques. 
57

58

59

60

61

62

63

64

65

66

67

68

\section{Proficient brain for optimal performance: The MAP model perspective}

This article is based on the distinction between performance effectiveness and processing efficiency (Eysenck \& Calvo, 1992; Eysenck, Derakshan, Santos, \& Calvo, 2007), and concerns the functioning of athletes during different types of optimal and suboptimal performance as conceived in the multi-action plan (MAP) model (Bortoli, Bertollo, Hanin, \& Robazza, 2012). Performance effectiveness has been defined as the quality of performance (e.g., the shooting scores). Processing efficiency regards the relationship between performance effectiveness and the use of resources or effort. According to Eysenck and Derakshan (2011), “...processing efficiency is high when performance effectiveness is high and use of resources is low and it is low when performance effectiveness is low but use of resources is high" (p. 956). However, this definition is not always tenable. Applied and theoretical studies observing the interaction between performance effectiveness and processing efficiency have shown that elite athletes can function in different modes when experiencing optimal performance (Bortoli et al., 2012; Bertollo et al., 2013; Carson \& Collins, 2016; Furley, Schweizer, \& Bertrams, 2015; Swann, Keegan, Crust, \& Piggott, 2016). Athletes are able to attain effective performances not only when they execute in automatic, fluent, and flow-like states, but also when they proficiently exert some effort to cope with distressful situations. Proficient athletes, indeed, are able to achieve performance goals or results with a high degree of certainty, minimum energy expenditure, and minimum movement time in different states and conditions (Magill \& Anderson, 2014). Proficiency is an individual's capability of achieving a particular and complex task by switching effectively between an automated and a more controlled execution according to the task and situational demands. 
According to this view, two qualitatively different modes of processing, namely,

80

81

82

83

84

85

86

87

88

automatic and controlled, have been recently proposed in the sport science field drawing on existing theories in general psychology (see Furley et al., 2015). Both types of performance are optimal, but Type 1 (fluent, automatic, and procedural) autonomous processing would be initiated and completed in the presence of relevant triggering conditions, whereas Type 2 (competent, regulated, and declarative) controlled processing would rely on working memory capacity to deal with novel problems, uncommon situations or unexpected events.

Referring to the performance effectiveness and processing efficiency distinction, optimal performance can be achieved in conditions of either efficient processing (i.e., fluent performance and low attentional control and/or effort) or effortful processing (i.e., high attentional control and/or effort). In others words, proficient athletes can complete the task successfully using both efficient and effortful types of processing (Wilson, 2008). In the sport domain, flow-like states in which athletes perform fluently are rare. Thus, athletes need to use their proficiency, developed through a large amount of deliberate practice (Ericsson, 2007), to maintain high performance levels and cope with distressful situations.

\section{Behavioural studies on performance effectiveness and processing efficiency}

The interplay between performance effectiveness and processing efficiency in elite performance has been investigated using distractions theories and self-focus theories to explain the anxiety-performance relationship (for a discussion, see Hill, Hanton, Matthews, \& Fleming, 2010).

Among the distractions theories (Eysenk, 1992; Eysenck, Derakshan, Santos, \& Calvo, 2007), attentional control theory (ACT) is concerned with attentional control in the context of anxiety and cognitive performance (Eysenk et al., 2007). It has also been applied to the motor 
102 behaviour and sport field to investigate how anxiety influences attention and performance in 103 goal-directed motor tasks (Coombes, Higgins, Gamble, Cauraugh, \& Janelle, 2009). Evidence

104 from the literature suggests that anxiety impairs processing efficiency more than performance 105 effectiveness (Coombes et al., 2009). Furthermore, a high level of state anxiety can impair both 106 processing efficiency and motor performance (e.g., Wilson, Wood, \& Vine, 2009). In ACT, the 107 crucial hypothesis is that anxiety will typically impair processing efficiency more than 108 performance effectiveness (Eysenck \& Derakshan, 2011). Taking a different perspective, self109 focus theories (explicit monitoring, Beilock \& Carr, 2001, and reinvestment, Master \& Maxell, 110 2008) centre on the excessive reinvestment of attention on movement execution. This conscious 111 control of the technique through attention 'reinvestment' leads to eventual breakdown in 112 performance and/or chocking under pressure. Recently, Carson and Collins (2016) have distinguished between positive and negative

114 self-foci in attention control, and argued that what and how performers direct their attention is 115 crucial to performance. This view concurs with the $2 \times 2$ conceptualization in the MAP model 116 (Bortoli et al., 2012), in which performance is classified in terms of both performance level 117 (optimal and suboptimal) and action control (automatized and controlled), as explained in greater 118 detail below.

119 Brain activity assessment of processing efficiency

120 In addition to the many behavioural studies reported in the literature, which support the

121 hypothesis that anxiety could impair processing efficiency more than performance effectiveness 122 (for a review, see Eysenck et al., 2007), there are several studies that have analysed processing 123 efficiency by using different techniques for brain activity assessment (Bishop, 2009; Righi, 124 Mecacci, \& Viggiano, 2009; Savostyanov et al., 2009). Findings showed that high levels of 
125 anxiety can be associated with greater brain activity compared to low levels, even when anxiety

126 has no impact on performance. Using the same psychophysiological and methodological

127 perspective, the present study focuses on the neural efficiency hypothesis as a framework to test

128 the relationship between performance effectiveness and the use of resources or effort during elite 129 performance in sport.

130 The neural efficiency hypothesis of psychomotor performance (see Del Percio et al., 131 2008; Hatfield \& Kerick, 2007) derives from studies on the relationship between brain and 132 intelligence, championed by Haier (Haier et al., 1988, 1992, 2004). Efficiency occurs as a result 133 of the disengagement of brain areas that are irrelevant for a given task, along with the 134 simultaneous engagement of highly task-relevant areas (Haier et al., 1992). In fact, extant 135 research supports the notion that EEG topographical oscillations in the theta and alpha band are 136 associated with cognitive performance (Klimesch, 1999, 2012). Overall, synchronization (ERS) 137 in the theta band has been linked to encoding of new information in the episodic memory due to 138 a task-related power increase, while the frontal midline theta has been associated with sustained 139 attention and top-down processing in precision sports (Chuang, Huang, \& Hung, 2013;

140 Doppelmeyr et al., 2008; Klimesch, 1999). De-synchronization (ERD) in the lower alpha band

141 (i.e., 8-10 Hz) is thought to reflect general task demands and attentional processes (i.e.,

142 vigilance, arousal), whereas desynchronization in the upper alpha band (i.e., 10-12 Hz) has been 143 associated with semantic performance (Klimesch, 1999) and task-related attention (Klimesch, 144 2012). Moreover, alpha ERS and ERD reflect inhibition and the release from inhibition, 145 respectively (Klimesch, 2012). In addition, excessive controlled processing impairs automaticity 146 (Masters \& Maxwell, 2008), and is linked to higher cortical activity in the attentional network, 147 especially in the frontal midline and parietal areas (Kao et al., 2013). 
In the sport sciences domain, neural efficiency has been paralleled to "the most efficient

149 movement" in terms of the energy cost or work output of a given movement, and has been

defined as psychomotor efficiency (Hatfield \& Kerick, 2007). High-skilled athletes usually

151 perform with minimal effort in comparison to novices. Noteworthy, Hatfield and Kerick (2007)

152 highlighted that economy of effort is a marker of superior psychomotor performance, thereby

153 supporting the notion that high-level performance is marked by economy of brain activity with

154 an inverse relationship between performance effectiveness and resources utilization.

155 Additional research on the neural efficiency hypothesis has corroborated the notion that

156 skilled performance in self-paced sports is usually accompanied by a lower and/or task-

157 synchronized cortical activity (Del Percio et al., 2009; Del Percio et al., 2010; Del Percio et al.,

158 2008). For instance, Babiloni and colleagues (2010) found greater resting alpha power in elite

159 athletes compared to amateur athletes and non-athletes. They concluded that athletes' brains are

160 more inhibited during resting states in agreement with the neural efficiency hypothesis. Also

161 congruent with the neural efficiency hypothesis, findings from a study by Del Percio and

162 colleagues $(2009,2011)$ suggest that elite athletes exhibit less alpha power reactivity than non163 athletes.

Altogether, neural efficiency seems to reflect two different processes in distinguishing elite from expert and novice athletes. "The first is a reduction in neural activity in certain brain regions as a particular skill becomes more automated and less controlled. The second is a

167 reduction of activity in sensory and motor cortex, reflecting more efficient processing made 168 possible by less energy expenditure" (Callan \& Naito, 2014, p. 183). Specifically, sensorimotor rhythms (12-15 Hz) are involved in visuo-motor tasks and may represent adaptive information 
170 processing during motor execution (Cheng et al. 2015a), sensitive to neurofeedback intervention

171 (Cheng et al. 2015b).

172 Although a number of studies support the neural efficiency hypothesis, there is emerging

173 evidence suggesting that this hypothesis does not fully account for elite athletes' brain activity in

174 self-paced sports (Vecchio, Del Percio, \& Babiloni, 2012). In particular, two recent experimental

175 studies in golf did not lend support to the neural efficiency hypothesis. In the first study, Babiloni

176 and colleagues showed that alpha ERD during complex visuo-motor integration was higher when

177 comparing athletes with non-athletes, and best performance with worst performance in elite

178 athletes (Babiloni et al., 2008). In the second study, they observed that successful putts of elite

179 golfers were related to strong parieto-central and parieto-frontal alpha coherence (Babiloni et al., 180 2011).

181 Of note, the neural efficiency hypothesis emerged mainly from studies comparing experts 182 and novices (i.e., expert-novice paradigm), and thus might not illustrate the unique neural 183 mechanisms associated with expert performance in sports (expert-performance approach), in the 184 sense that it does not explain the interplay between processing efficiency and performance 185 effectiveness. Therefore, the unique neural mechanisms associated with optimal and suboptimal performance states among skilled athletes remain an understudied topic (Comani et al, 2014;

187 Bertollo et al, 2013; di Fronso, Robazza, Filho, Bortoli, Comani, \& Bertollo, 2016). Put 188 differently, it is established that experts will show a greater degree of automaticity and neural efficiency when compared to novices (Callan \& Naito, 2014; Del Percio et al., 2008). However, it remains mostly unknown whether and how neural efficiency among brain cortices is related to different performance states among highly skilled athletes. 
mechanisms underlying optimal and suboptimal performance states among high-skilled athletes,

Bortoli and colleagues (2012) have developed the MAP intervention model. The model is

embedded within the IZOF framework (Hanin, 2007), and fits well with the ACT hypothesis that

concurs with dual-process theories that describe and explain the dual modes of processing of

athletes, namely, automatic and controlled (see Furley et al., 2015). In this view, Type 1

automatic processing allows for the fast and effortless execution of behavioural responses,

whereas Type 2 controlled processing is well equipped for dealing with novel problems or

skill establishment is related to aspects governing motor control. From this viewpoint, skill

establishment concerns the structures/representations of the movement and refers to the level and consistency of automaticity in the execution of different movement components (Carson \& Collins, 2016).

According to the MAP model (Bortoli et al., 2012), performance experience can be depicted along two different axes: one represents the performance level per se, and the other represents the amount of control over the task. According to this interaction, this performance experience plane can be classified into four performance Types: optimal-automatic (Type 1), optimal-controlled (Type 2), suboptimal-controlled (Type 3), and suboptimal-automatic (Type 4;

212 see Figure 1). In Type 1 performance (flow condition) the athlete is self-confident and in control

213 of the situation, and is able to direct his or her physical and mental energies toward the

214 accomplishment of a task. This type of processing is independent of available working memory 
216 play a role of supervision. Both performance effectiveness and processing efficiency are high.

217 Stress, fatigue, unexpected events, or performance issues could disrupt performance. These

218 unfavourable conditions can lead to Type 3 performance, typified by an excessive reinvestment

219 in controlled processing and an overload of working memory, which undermines movement

220 fluidity and automaticity (Maxwell, Masters, \& Eves, 2000; Oudejans, Kuijpers, Kooijman, \&

221 Bakker, 2011). Both performance effectiveness and processing efficiency are low. Finally, Type

2222 performance state is characterized by high performance effectiveness and low processing

223 efficiency (i.e., effortful processing and involvement of working memory; Eysenck \&

224 Derakshan, 2011), while Type 4 performance is typified by ineffective performance, inefficient

225 processing, and no reliance on working memory.

226 Research findings on the MAP model have shown that different physiological (e.g., skin

227 conductance responses, breathing rate, and heart rate) and behavioural markers (e.g., kinematic

228 patterns) underlie the four types of performance states (Bertollo et al., 2013; Filho et al., 2015)

229 and focus of attention can affect performance (Bertollo et al., 2015). There is also initial

230 evidence that different neural patterns are associated with the four performance types foreseen in

231 the MAP model (Comani et al., 2014; di Fronso et al., 2016). Specifically, Comani et al. (2014)

232 observed that optimal-automatic performance (i.e., Type 1) states among shooters were

233 characterized by lower alpha power in the central, contralateral parietal, and occipital areas (at

234 shot release), in agreement with the neural efficiency hypothesis (i.e., global decrease in cortical

235 activity). Conversely, optimal-controlled performance (i.e., Type 2) was characterized by an

236 increased alpha power activity in the frontal and occipital areas.

237 


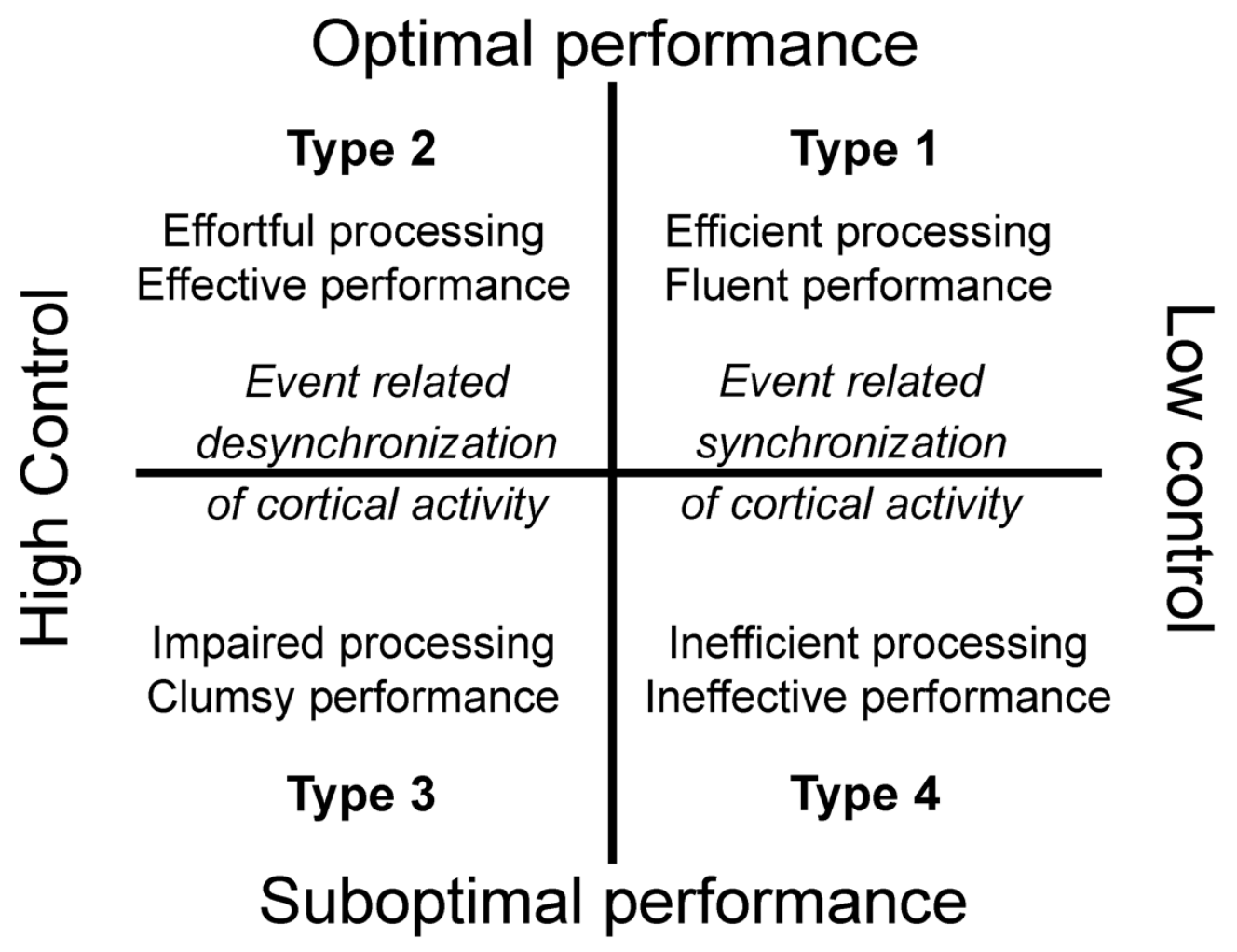

Figure 1. The multi-action plan model.

In the present study, we aimed to further explore the neural dynamics associated with

optimal/suboptimal by controlled/automated performance states among high-skilled athletes in

accordance with the MAP model tenets. We were particularly interested in examining the neural

activity associated with optimal-automated (Type 1) and optimal-controlled (Type 2)

performances in the light of the reviewed literature on ACT theory, neural efficiency, and dual-

248 process theories in sport and motor performance. According to the MAP model assumptions and

249 previous research findings, we expected to find different patterns of cortical activity associated 
250 with the four types of performance states. In particular, we expected to observe more cortical

251 synchronization in Type 1 and Type 4 performance states (see Figure 1). This hypothesis relies

252 on earlier findings that showed some similar psychophysiological features underlying Type 1 and

253 Type 4 performances (Bertollo et al., 2013, di Fronso et al., 2016, Robazza, Bertollo, Filho,

254 Hanin, \& Bortoli, 2016). On the other hand, we expected to observe more cortical

255 desynchronization associated with Type 2 and Type 3 performance states. Specifically, we

256 expected to find the following cortical patterns:

257 1. Type 1 performance: (a) greater ERS in the theta band reflecting the successful encoding

258 of information and the activity of the attentional network; (b) at the same time, ERS activity in both low and high alpha band, which is an expression of the inhibition processes (Klimesch, 2012) and neural efficiency (Del Percio et al., 2008).

2. Type 2 performance: (a) an ERS activity similar to Type 1 performance, with more ERD in the low and high alpha band, reflecting the two faces of inhibition, namely, selective activation vs. blocking of information processing; (b) more selective activation related to the attentional control exerted on the action.

3. Type 3 performance: (a) a clear ERD pattern in the theta band, indicating specific attention to the task at hand. Indeed, theta response over frontal regions reflects the activation of neural networks involved in the allocation of attention related to target stimuli: a parietal activation would occur during the processing of visuospatial information, and a premotor cortex activation would occur during visuo-motor processing; (b) more ERD in the low alpha band associated with higher spread attention engaged and effort resources utilization; (c) more ERD in the areas associated with verbal semantic processes or visuo-spatial processing. 
273

274

275

276

277

278

279

280

281

282

283

284

285

286

287

289

290

291

292

293

294

295

4. Type 4 performance: (a) a cortical activity pattern similar to Type 1 performance; (b) a hyper-synchronization (high ERS) during the entire interval preceding execution because of low controlled and disengaged behaviour with no reliance on working memory. It is well known that hyper-synchronization is associated with no reliance on working memory and a sort of loss of consciousness derived from task disengagement (Klimesch, 2012).

\section{Method}

\section{Participants}

Ten elite, right-handed shooters (6 male and 4 female), with extensive experience in international competitions (e.g., World Championships, World Cup Championships, Olympic Games) and included in the International Shooting Sport Federation international ranking, participated in the study. The participants ranged in age from 18 to 29 years $(M=22.8, S D=3.5)$ and had at least 10 years of experience $(M=14.5, S D=4.0)$. After being briefed on the general purpose of the study, the participants agreed to participate and signed a written informed consent. The study was conducted in accordance with the declaration of Helsinki and received approval from the local university ethics committee (ref. n. 10-21/05/2015).

\section{Procedure}

The participants were asked to identify the core components of their "chain of action" that were essential for optimal performance. They were then asked to choose one element (i.e., an idiosyncratic core component, such as aiming, triggering, and front sight alignment) deemed fundamental in order to perform optimally according to the procedure developed by Bortoli et al. (2012). Subsequently, the participants performed a total of 120 air-pistol shots. They were "free to relax" between shots and to shoot when they felt "ready to go" (average inter-shot interval of 
296 about 1 minute). The distance between the shooter and the target was $10 \mathrm{~m}$, and the diameter of

297 the target was $6 \mathrm{~cm}$, in accordance with international shooting competition rules (www.issf-

298 sports.org/theissf/rules/english_rulebook.ashx). An electronic scoring target (Hs10 Hybridscore,

299 SIUS, Effretikon, Switzerland) was used to (automatically) record the shooting scores. Shooting

300 score results were initially concealed from the athletes as we were interested in assessing their

301 perceived accuracy. Hence, after each shot, the athletes were asked to report their perceived

302 shooting score (ranging from 0 to 10.9). They also reported the control level of the idiosyncratic

303 core component of action (from 0 to 11 on a Borg scale; for a detailed description see Bertollo et

304 al., 2013). After this evaluation, the actual shooting score (i.e., objective performance) of each

305 shot was made available to the shooter. In addition to self-evaluation, we also recorded their

306 brain waves using a 32-channel waveguard cap by ANT (Advanced Neuro Technology, Enshede,

307 The Netherlands).

308 EEG recordings. EEG data were continuously recorded (sampling frequency: 1024 Hz)

309 from the 32 scalp electrodes (active electrodes for movement compensation) positioned over the

310 scalp according to the 10-20 system, using an eegosport ${ }^{\mathrm{TM}}$ amplifier (ANT, Enschede,

311 Netherlands). EEG signals were recorded with common reference. The ground electrode was

312 positioned between Fpz and Fz, and the electrode impedance was kept below $10 \mathrm{k} \Omega$. A device

313 based on acoustic technology (cardio-microphone and Powerlab 16/30, ADInstruments,

314 Australia) was used to identify the instant of shot release. It was positioned on a tripod $30 \mathrm{~cm}$

315 from the air pistol and the acoustic signals were acquired with a sampling frequency of $1 \mathrm{kHz}$.

316 Performance categorization. The participant' shooting scores and perceived control

317 levels were used to categorize the EEG epochs into a $2 \times 2$ matrix using the median split

318 technique to identify the four types of performance as defined in the MAP model. Following this 
319 approach, shooting results from 10.2 to 10.9 (maximal possible score) were categorized as

320 optimal, and the remaining scores as suboptimal. Attentional control levels $\leq 4$ were categorized

321 as automatic performance and the others as controlled performance. Therefore, the combination

322 of shooting result $>10.2$ and control level $\leq 4$ led to a performance categorization as optimal-

323 automatic, i.e. as Type 1, whereas the performance was classified as optimal-controlled, i.e. Type

3242 with the combination of shooting result $>10.2$ and control level $>4$,. When the shooting result

325 was $<10.2$ and the control level was $>4$, performance was considered as suboptimal-controlled,

326 i.e. Type 3, whereas performance was classified as suboptimal-automatic, i.e. Type 4 when the

327 shooting result was $<10.2$ and the control level was $\leq 4$.

EEG data pre-processing. EEG data were band-pass filtered between 0.3 and $40 \mathrm{~Hz}$, and segmented into single epochs of $10 \mathrm{~s}$ duration. Each epoch started at $-6 \mathrm{~s}$ and ended at $+4 \mathrm{~s}$ with respect to shot release $(\mathrm{t}=0)$. Data epochs were visually inspected, and those showing instrumental, ocular and muscular artefacts were corrected using the tool available in the ASA software (Zanow \& Knosche, 2004). The data epochs showing residual artefacts were excluded from further analysis.

ERD/ERS analysis. We were particularly interested in the relationship between attention and performance. Thus, we ran the ERD/ERS analysis in the theta, and low and high alpha frequency bands. To quantify the event-related changes in these frequency bands, the individual ERD/ERS maps were calculated following the procedure proposed by Zanow and Knosche (2004), where ERD and ERS are defined, respectively, as the percent increase and decrease of signal power as compared to the baseline. This definition is different from that proposed by Pfurtscheller and da Silva (1999). Following the procedure proposed by Zanow and Knosche 341 (2004), the Hilbert transform was performed before ERD/ERS analysis. For a given frequency 
342 band, ERD/ERS maps were calculated for an interval of interest as the percent variation of the

343 signal power with respect to baseline values for each EEG channel.

Given that the individual alpha frequency was similar across participants (9.9 to $10.1 \mathrm{~Hz})$,

345 we considered the following frequency bands: $4-8 \mathrm{~Hz}$ (i.e., theta), $8-10 \mathrm{~Hz}$ (low alpha), and 10-

$34612 \mathrm{~Hz}$ (high alpha) for analysis. Three intervals of interest, each of $1 \mathrm{~s}$ duration, were considered

347 during the $3 \mathrm{~s}$ preceding each shot (i.e., from -3 to 0 ), whereas the baseline signal was windowed

348 from -5 to $-4 \mathrm{~s}$ before the shot. Periods before $-5 \mathrm{~s}$ were not suitable for analysis because of body

349 movements, small adjustments of the head/trunk, and respiration. For each participant and for

350 each group of EEG epochs categorized according to the types of performance foreseen in the

351 MAP model, the baseline signals were averaged to reduce background noise before ERD/ERS

352 calculation. Similarly, averaged ERD/ERS maps were obtained from the single ERD/ERS maps

353 for each interval of interest, for each participant and for each group of EEG epochs. Finally, for

354 each type of performance the individual ERD/ERS maps were averaged across subjects to

355 account for the cortical patterns underlying the four MAP model types, thus obtaining average

356 topographical maps.

Statistical Analysis. The percentage of ERD/ERS individual data was exported with

358

359

360

361

362

363

364
ASA software (ANT-Neuro) and analysed using Statistica 10 software (Statsoft). Separated repeated measures analyses of variance (RM-ANOVAs) for each electrode were applied to each frequency band (theta, low alpha, and high alpha). Huynh-Feldt correction was applied (Huynh \& Feldt, 1976) when the assumption of sphericity was violated. 


\section{Results}

Descriptive statistics for the behavioural data for each performance type as derived from

367

368

369

370

371

372

373

\section{4}

375

376

377

378

379

380

381

382

383

the interaction of optimal/suboptimal and automatic/controlled performance dimensions are

summarized in Table 1.

Table 1. Mean and standard deviation during each performance type of shooting result and control level on the core component of the action. * The number of shots for each performance type derives from the number of trials (120) for each athlete (10) without the shots affected by EEG artefacts.

\begin{tabular}{cccc}
\hline Performance Type & $\begin{array}{c}\text { Number of } \\
\text { Shots* }\end{array}$ & $\begin{array}{c}\text { Shooting } \\
\text { Results }\end{array}$ & $\begin{array}{c}\text { Control Level on } \\
\text { Core Components }\end{array}$ \\
\hline 1 & 238 & $10.51(0.12)$ & $4,59(0.39)$ \\
2 & 350 & $10.45(0.12)$ & $6.47(0.54)$ \\
3 & 212 & $9.90(0.19)$ & $6.44(0.46)$ \\
4 & 348 & $9.76(0.24)$ & $4.39(0.54)$ \\
\hline
\end{tabular}

A $4 \times 3$ (performance types $\times$ time) repeated measures analysis of variance $(\mathrm{RM}-$

ANOVA) was performed to test the differences among the four types of performance during the three seconds preceding the shots for theta, low alpha and high alpha frequency bands. The dependent variables were the ERD/ERS percentages in each frequency band (i.e., theta, low alpha, high alpha) for each electrode site across the scalp. This analysis was conducted on approximately 90 shots for each participant in three different frequency bands.

\section{Theta ERD/ERS}

A series of RM-ANOVAs $4 \times 3$ (performance $\times$ time) on the ERD/ERS theta band yielded significant effects on performance, time, and the interaction performance by time. Only the significant results are reported in Table 2. 
387 Table 2. Significant results of the RM-ANOVAs $4 \times 3$ (performance $\times$ time) on the ERD/ERS theta band

\begin{tabular}{lccccc}
\hline \multicolumn{7}{c}{ Theta ERD/ERS } \\
\hline Variable & Electrodes & Degrees of freedom & $\mathbf{F}$ & $\boldsymbol{p}$ & $\boldsymbol{\eta}_{\mathbf{p}}{ }^{\mathbf{2}}$ \\
\hline Performance & Fpz & 3,27 & 5.338 & .005 & .372 \\
Performance & FC2 & 3,27 & 4.230 & .014 & .320 \\
Performance & CP2 & 3,27 & 4.054 & .016 & .311 \\
Performance & CP6 & 3,27 & 3.222 & .038 & .264 \\
Time & F7 & 2,18 & 5.005 & .018 & .357 \\
Time & FC1 & 2,18 & 5.317 & .015 & .371 \\
Time & FC5 & 2,18 & 7.316 & .004 & .410 \\
Time & T7 & 2,18 & 7.888 & .003 & .467 \\
Time & Cz & 2,18 & 4.054 & .035 & .311 \\
Time & CP1 & 2,18 & 8.858 & .001 & .496 \\
Time & CP5 & 2,18 & 3.972 & .037 & .306 \\
Performance x Time & Fz & 6,54 & 2.033 & .047 & .204 \\
Performance x Time & CP6 & 6,54 & 2.680 & .023 & -229 \\
\hline
\end{tabular}

388

Figure 2 illustrates the grand averages of the scalp topographical distributions of the release $(\mathrm{t}=0)$. results in theta band are reported in the supplemental materials. 


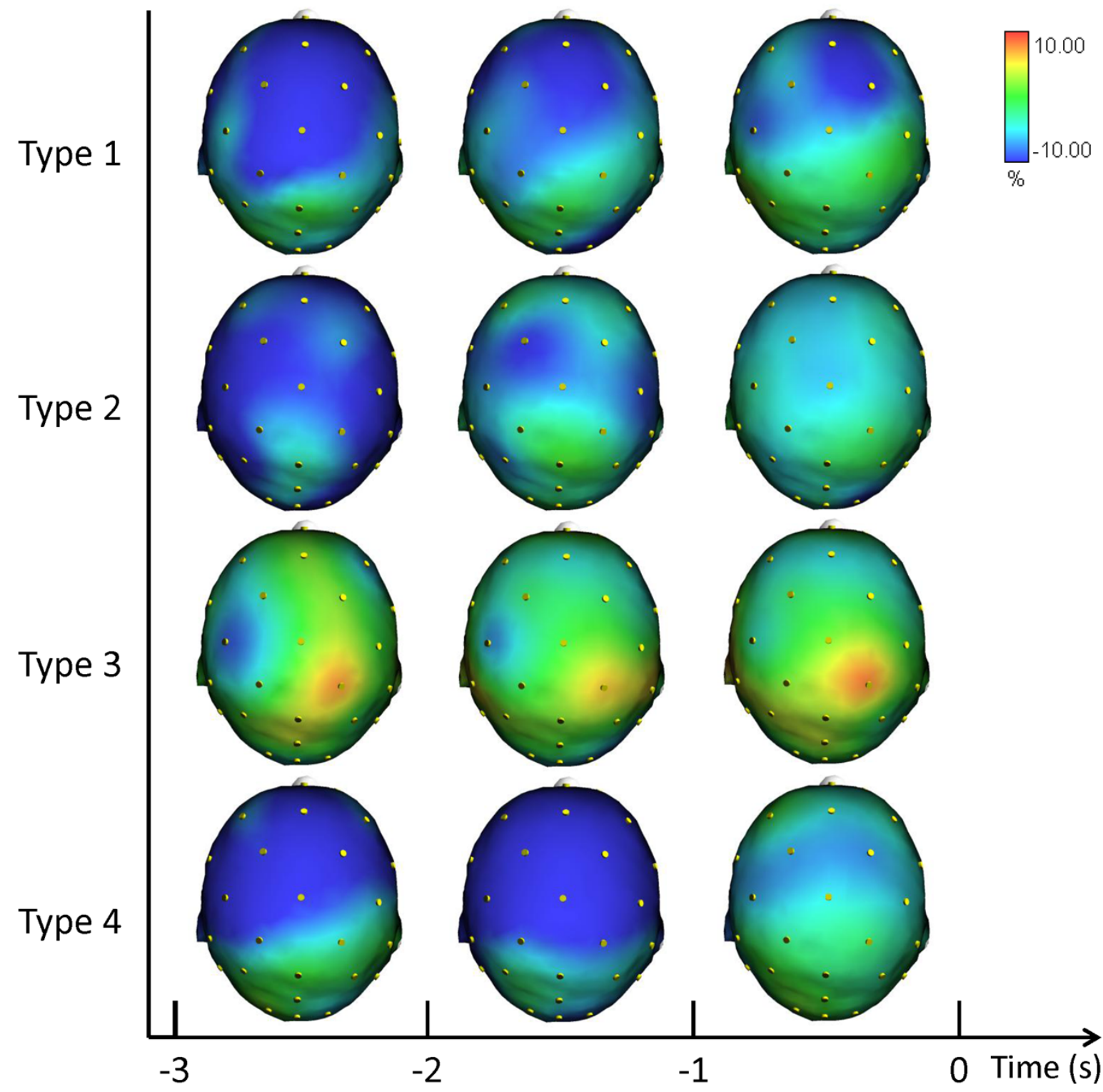

Figure 2. Average topographical distributions of the ERD/ERS amplitudes in the theta band for each performance type during the $3 \mathrm{~s}$ before shot release. The theta ERD/ERS maps were calculated for three pre-shot periods: from $-3 \mathrm{~s}$ to $-2 \mathrm{~s}$, from $-2 \mathrm{~s}$ to $-1 \mathrm{~s}$, and from $-1 \mathrm{~s}$ to shot release $(\mathrm{t}=0)$. Time scale is on the $\mathrm{X}$ axis. Color scale: maximum ERD and ERS are coded in red and blue, respectively. The maximal (\%) value of the ERD/ERS is given at the top right side of the figure. 
406 yielded significant effects on performance and time. The significant results are reported in Table

407 3. No differences on the performance by time interaction were found.

408

409 Table 3. Significant results of the RM-ANOVAs $4 \times 3$ (performance $\times$ time) on the ERD/ERS 410 low alpha band.

\begin{tabular}{lccccc}
\hline \multicolumn{7}{c}{ Low Alpha ERD/ERS } \\
\hline Variables & Electrodes & Degrees of freedom & $\mathbf{F}$ & $\boldsymbol{p}$ & $\boldsymbol{\eta}_{\mathbf{p}}{ }^{\mathbf{2}}$ \\
\hline Performance & $\mathrm{F} 8$ & 3,27 & 3.279 & .040 & .261 \\
Performance & $\mathrm{FC} 2$ & 3,27 & 5.105 & .006 & .358 \\
Time & $\mathrm{Fp} 1$ & 2,18 & 3.969 & .048 & .306 \\
Time & $\mathrm{Fpz}$ & 2,18 & 4.635 & .044 & .340 \\
Time & $\mathrm{F} 7$ & 2,18 & 8.905 & .002 & .497 \\
Time & $\mathrm{F} 3$ & 2,18 & 3.767 & .042 & .295 \\
Time & $\mathrm{F} 4$ & 2,18 & 8.809 & .002 & .495 \\
Time & $\mathrm{F} 8$ & 2,18 & 3.845 & .040 & .299 \\
Time & $\mathrm{FC} 5$ & 2,18 & 8.879 & .002 & .497 \\
Time & $\mathrm{FC} 1$ & 2,18 & 4.504 & .025 & .334 \\
Time & $\mathrm{T} 7$ & 2,18 & 5.544 & .028 & .381 \\
Time & $\mathrm{C} 3$ & 2,18 & 9.101 & .001 & .503 \\
Time & $\mathrm{Cz}$ & 2,18 & 6.124 & .009 & .405 \\
Time & $\mathrm{Cp} 5$ & 2,18 & 6.193 & .008 & .408 \\
Time & $\mathrm{Cp} 1$ & 2,18 & 10.926 & .001 & .548 \\
Time & $\mathrm{Oz}$ & 2,18 & 5.842 & .011 & .394 \\
Time & $\mathrm{O} 2$ & 2,18 & 5.554 & .013 & .382 \\
\hline
\end{tabular}

411

In Figure 3 we plotted the grand averages of the scalp topographical distributions of the

ERD/ERS amplitudes in the low alpha frequency band for each performance type. The low alpha

415 to shot release $(\mathrm{t}=0)$. Similiarly to the theta band results, we performed the post hoc analysis for

416 performance and performance by time interaction. Fisher post-hoc analyses for the significant

417 results in low alpha band are included in the supplemental materials. 


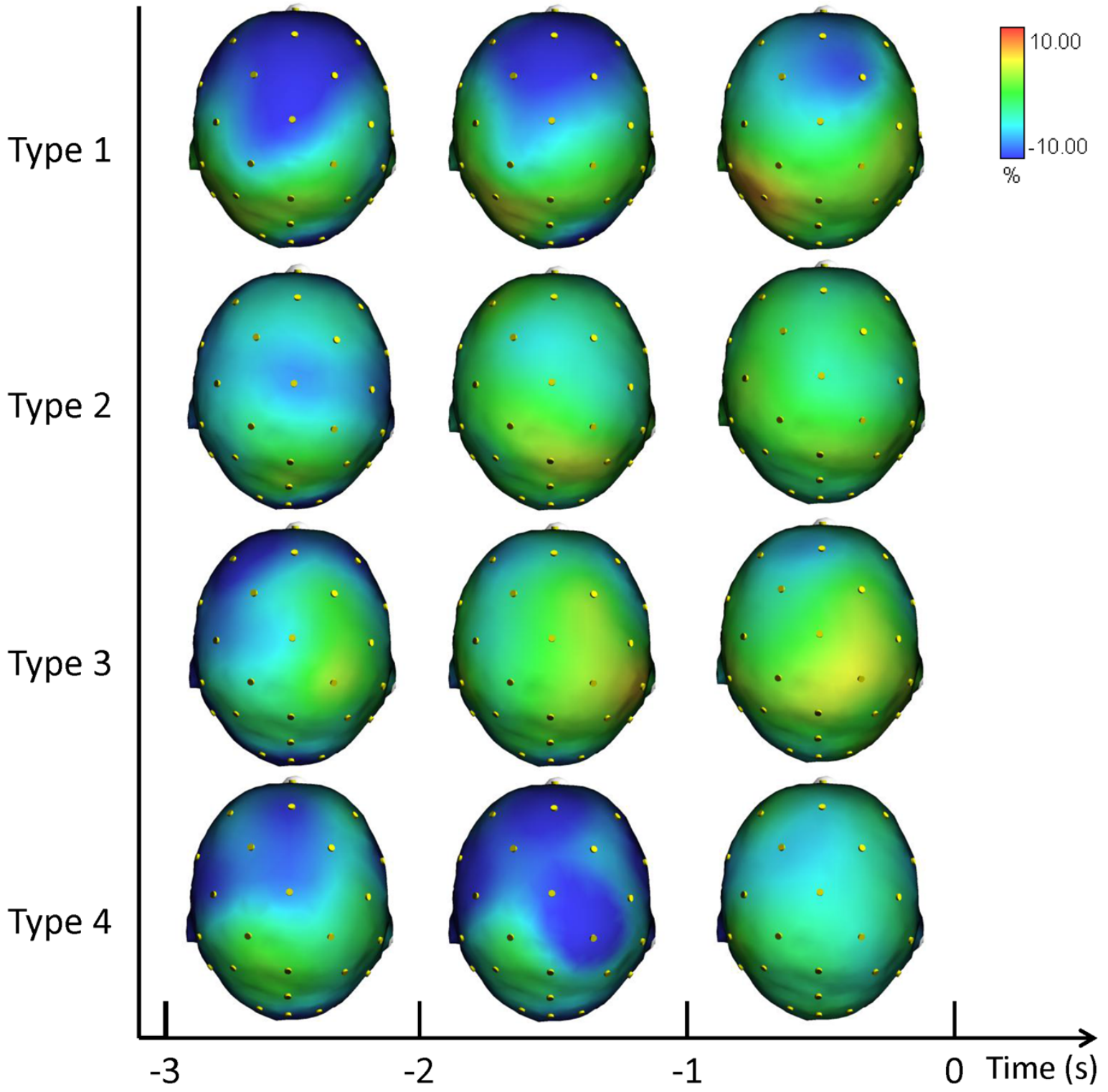

Figure 3. Average topographical distributions of the ERD/ERS amplitudes in the low alpha frequency band for each performance type during the $3 \mathrm{~s}$ before shot release. The low alpha ERD/ERS maps were calculated for three pre-shot periods: from $-3 \mathrm{~s}$ to $-2 \mathrm{~s}$, from $-2 \mathrm{~s}$ to -1 $\mathrm{s}$, and from $-1 \mathrm{~s}$ to shot release $(\mathrm{t}=0)$. Time scale is on the $\mathrm{X}$ axis. Colour scale: maximum ERD and ERS are coded in red and blue, respectively. The maximal (\%) value of the ERD/ERS is given at the top right side of the figure. 
428 yielded significant effects on performance, time, and the performance by time interaction. The

429 significant results are reported in Table 4.

430

431 Table 4. Significant results of the RM-ANOVAs 4 x 3 (performance x time) within subjects on 432 the ERD/ERS high alpha band.

\begin{tabular}{|c|c|c|c|c|c|}
\hline \multicolumn{6}{|c|}{ High Alpha ERD/ERS } \\
\hline Variables & Electrodes & Degrees of freedom & $\mathbf{F}$ & $p$ & $\eta_{p}^{2}$ \\
\hline Performance & Fp1 & 3,27 & 3.570 & .026 & .284 \\
\hline Performance & F8 & 3,27 & 3.038 & .046 & .252 \\
\hline Performance & P3 & 3,27 & 3.721 & .023 & .292 \\
\hline Time & Fp1 & 2,18 & 5.460 & .029 & .378 \\
\hline Time & Fp2 & 2,18 & 4.811 & .040 & .348 \\
\hline Time & F7 & 2,18 & 4.670 & .049 & .342 \\
\hline Time & $\mathrm{F} 4$ & 2,18 & 8.354 & .002 & .481 \\
\hline Time & $\mathrm{Fc} 5$ & 2,18 & 8.186 & .002 & .476 \\
\hline Time & $\mathrm{Fc} 2$ & 2,18 & 4.846 & .020 & .350 \\
\hline Time & $\mathrm{T} 7$ & 2,18 & 4.813 & .021 & .348 \\
\hline Time & $\mathrm{C} 3$ & 2,18 & 4.689 & .022 & .343 \\
\hline Time & $\mathrm{Cz}$ & 2,18 & 4.322 & .029 & .329 \\
\hline Performance $\mathrm{x}$ Time & Fp1 & 6,54 & 2.968 & .033 & .248 \\
\hline Performance $\mathrm{x}$ Time & $\mathrm{Fz}$ & 6,54 & 2.401 & .039 & .211 \\
\hline Performance $\mathrm{x}$ Time & F8 & 6,54 & 3.196 & .009 & .262 \\
\hline Performance $\mathrm{x}$ Time & $\mathrm{C} 3$ & 6,54 & 3.473 & .005 & .224 \\
\hline Performance $\mathrm{x}$ Time & $\mathrm{Cp} 1$ & 6,54 & 2.401 & .039 & .278 \\
\hline
\end{tabular}

433

Figure 4 shows the grand averages of the scalp topographical distributions of the high

435 ERD/ERS amplitude in the alpha frequency band for each performance type. The high alpha

436 ERD/ERS was mapped during three pre-shot periods: from $-3 \mathrm{~s}$ to $-2 \mathrm{~s}$, from $-2 \mathrm{~s}$ to $-1 \mathrm{~s}$, and

437 from $-1 \mathrm{~s}$ to zero time. 

alpha band are included in the supplemental materials.

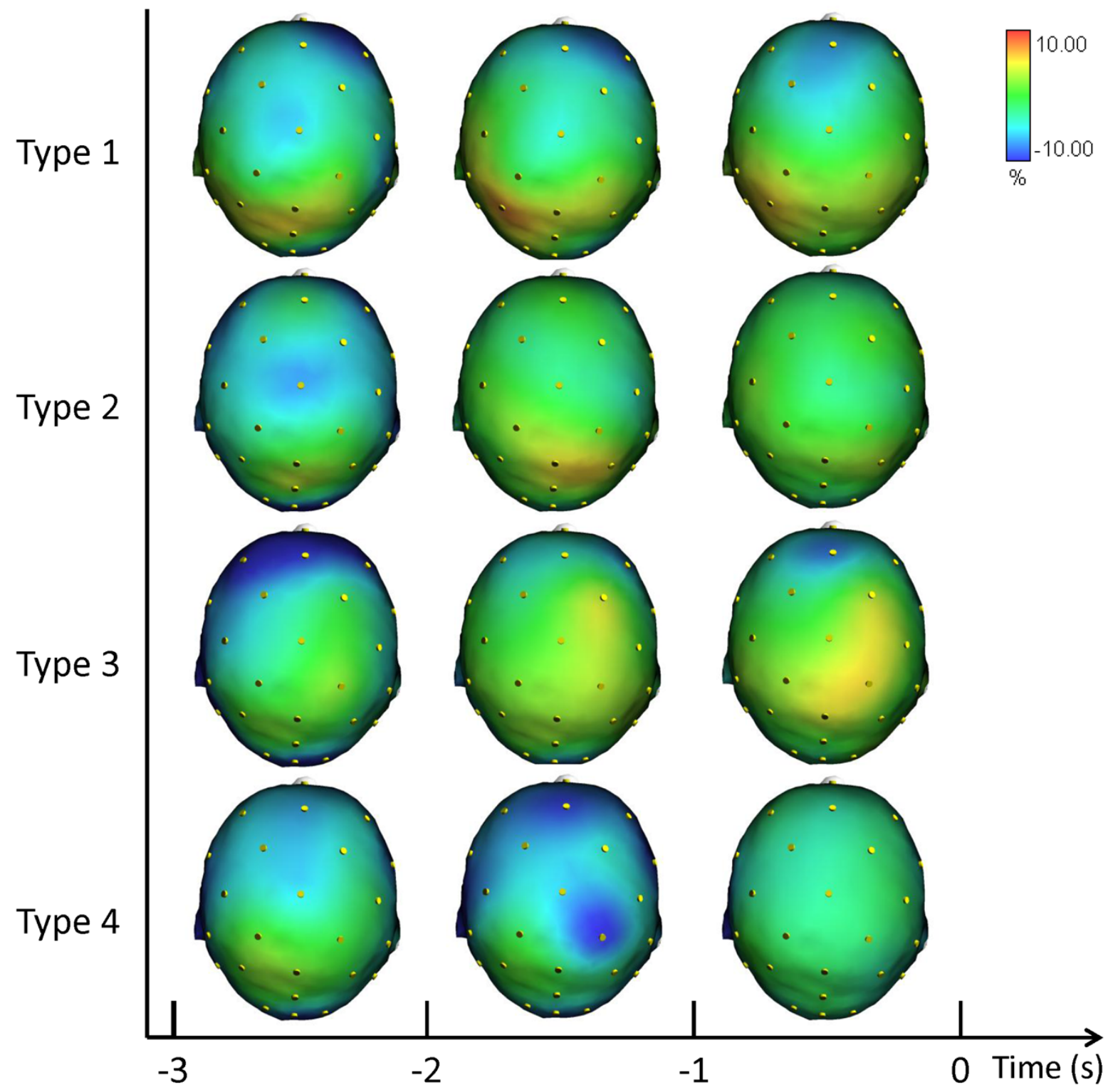

Figure 4. Average topographical distributions of the ERD/ERS amplitudes in the high alpha

ERD/ERS maps were calculated for three pre-shot periods: from $-3 \mathrm{~s}$ to $-2 \mathrm{~s}$, from $-2 \mathrm{~s}$ to $-1 \mathrm{~s}$, and from $-1 \mathrm{~s}$ to shot release $(\mathrm{t}=0)$. Time scale is on the $\mathrm{X}$ axis. Colour scale: maximum ERD and ERS are coded in red and blue, respectively. The maximal (\%) value of the ERD/ERS is given at 


\section{Discussion}

450

In the present study, we used a mobile EEG device to analyse the cortical activity

451

452

453

454

455

456

457

458

459

460

461

462

463

464

465

466

467

468

469

470

471

underlying different types of optimal and suboptimal performance states in the framework of the

MAP model. Assessing brain activity using mobile EEG data collection enables evaluation of neural efficiency during performance in actual settings (Park, Fairweather, \& Donaldson, 2015).

Our findings showed that elite athletes used different types of functioning to achieve optimal performance, sometimes typified by effort, attentional control, and use of resources higher than in flow-like automatic performance (Bortoli et al., 2012; Carson et al., 2016; Furley et al., 2015). This outcome is in line with the view that "the processing inefficiency caused by the disruption of the inhibition and shifting functions of the central executive system does not necessarily lead to decrements in performance effectiveness... by using compensatory or alternative processing strategies" (Wilson, 2008, p. 195). Similar behaviour has been observed when people have to cope with affordances that are not always appropriate in a particular situation, and therefore need to be inhibited by higher cognitive functions located in the frontal lobes (Furley et al., 2015). In brain studies, ERD has been associated with higher involvement of cognitive processes (in particular working memory), and reflects anticipatory attention (Klimesch, 2002; Nussbaumer, Grabner, \& Stern, 2015).

In the sport science domain, there is emerging evidence suggesting that the neural efficiency hypothesis does not fully account for elite athletes' brain activity (Vecchio et al., 2012). Vecchio and colleagues have argued that information processing in the cognitive-motor cortical systems of elite athletes is more complex than that predicted by the "neural efficiency" hypothesis. In experts, some neural networks within the cortex might reflect the "neural efficiency" (e.g., default mode network and relaxation), whereas other networks might reflect 
472 other mechanisms (e.g., task request, attentional demands, or affective demands). In cognitive

473 neuroscience, Poldrack (2014) recently raised doubts about efficiency as a useful concept to

474 explain expert performance. He proposed that the common usage of the concept of "efficiency"

475 in cognitive neuroscience is equally vacuous. In general, the term is used to describe situations

476 where performance appears similar but activation is greater for one group (e.g,. novices, which

477 are usually labelled as "less efficient").

478 In discussing the brain studies associated with superior performance, we adopted the

479 idiosyncratic framework of the MAP model to test different processing modes underpinning

480 different types of performance. In this framework, we hypothesized to integrate the neural

481 efficiency hypothesis within a broader concept, namely, the neural proficiency hypothesis of

482 superior performance, in which athletes' brain waves are modulated by the individual's effort to

483 maintain a high performance level by switching proficiently between Type 1 and Type 2

484 performance states. Proficiency is an active and qualitative process that involves the interaction

485 between the efficiency of human processing and the efficacy of the performance, and it is not

486 only the ratio between the input and the output of the biomechanical system to reach the

487 outcome. A proficient brain is the ensemble of the neural features that define the state of being

488 adequate or well qualified both physically and psychologically for the task. This implies that

489 efficient processing and inefficient processing (measured through cortical activity) during

490 performance are modulated by the degree of effort and by the attentional demands of the control

491 systems involved in the task. This is mirrored in the neural activity found in optimal (Type 1 and

492 Type 2) and suboptimal (Type 3 and Type 4) performance states, as found in earlier preliminary

493 case studies with elite athletes (Comani et al., 2014; di Fronso et al., 2016). Our findings are also

494 in line with a study on movement-related cortical activity as a function of expertise and pressure 
495 (Cooke et al., 2014), in which a greater reduction in EEG high-alpha power during preparation

496 for action was deemed to reflect more resources being devoted to response programming.

497 Indeed, both cortical excitatory and inhibitory factors can contribute to elite skilled performance

498 during different types of good and poor performances. This most likely depends on the different

499 degree of attentional requests on the task and the high anxiety levels usually found in

500 competition that can undermine automaticity (Hatfield et al., 2013). In this situation, a Type 2

501 processing, well equipped for dealing with novel problems, is more appropriate (Furley et al., 502 2015).

503 In agreement with prior studies demonstrating that the event-related changes of the 504 cortical desynchronization/synchronization (ERD/ERS) can capture differences in the brain 505 activity of elite athletes during optimal and suboptimal performances (Comani et al., 2014; Del 506 Percio et al., 2009; di Fronso et al., 2016). In the present study we investigated the event-related 507 changes of cortical activity (ERD/ERS) in the theta and alpha bands in association with different 508 performance states. ERS gathers a similar state of neuronal assemblies resulting in summation of 509 postsynaptic potentials (synchrony) due to similarity of neuronal states. Conversely, a dissimilar 510 state of neuronal assemblies results in differential assignment of neurons and de-synchrony or 511 changing power or amplitude (ERD). These two types of brain configurations are an expression 512 of the two classical types of performance: good and poor (Cooke, 2013; Del Percio et al., 2009; 513 Hatfield \& Kerick, 2007). In effect, the cortical patterns displayed in Figures 2, 3, and 4 confirm 514 that neural desynchronization (i.e., ERD) is mainly associated with Type 3 performance 515 (suboptimal-controlled). However, ERS activity was not only associated with optimal-automated 516 performance (Type 1), but also associated with suboptimal-automated performance (Type 4). In 517 addition, during optimal-controlled performance (Type 2) cortical activity showed greater ERD 
518 activity in different areas and frequency bands compared to optimal-automated performance,

519 contrary to the neural efficiency hypothesis, which states that more synchronization would

520 appear in association with good performance. However, when we observed a more diffused and

521 evident ERD activity in the brain (i.e., when athletes were unable to maintain a consistent

522 execution associated with economy of effort), we simultaneously observed a decline toward a

523 suboptimal-controlled performance (Type 3). This pattern is more evident in the theta band and it

524 involves the right parietal areas, in agreement with the evidence that (1) theta activity is also

525 present at parietal sites in the high- vs. low-error condition (Arrighi et al., 2016), and that (2)

526 integration of visual and spatial information involves both frontal and parietal areas (Morgan et

527 al., 2013).

528 The ability to achieve performance goals with a high degree of certainty, and not only

529 minimum energy expenditure, is paramount for high performance in sport. Thus, athletes need to

530 use different strategies to maintain or achieve good performance (Bortoli et al., 2012; Furley et

531 al., 2015; Swann et al., 2016). These strategies, that may involve the engagement of working

532 memory, require more effort than during optimal-automatic performance experiences (Type 1).

533 Processing efficiency tends to decrease during this type of effective performance (Type 2).

534 Indeed, Type 2 performance of our shooters was typified by a dissimilar state of neuronal

535 assemblies during active task engagement, resulting in differential assignment of neurons and

536 desynchronized theta/alpha power. Specifically, we found a significant desynchronization in the

537 visuo-spatial and visuo-motor attentional networks (Fpz Fc2 Cp2 Cp6) in the theta band during

538 Type 3 performance, whereas the other types of performance were still synchronized. These

539 findings also suggest that more sustained attention on the target associated with top-down

540 processes was necessary during execution (Kao, Huang, \& Hung, 2013; Missonnier et al., 2006). 
541 This is apparent from the results of the performance by time interaction in which we found

542 significant ERD in the frontal midline theta $(\mathrm{Fz})$ during the second before shot release as well as

543 de-synchronization in parietal areas (Cp6) two seconds before shot release, consistent with

544 findings from other studies (Chuang et al., 2013; Doppelmeyr et al., 2008; Kao et al., 2013).

545 Previous studies have found that neural networks engaged in the control of action are

546 dynamically reorganized depending on whether the task calls for volitional control or is

547 performed automatically (see Matsuzaka, Akiyama, \& Mushiake, 2013). Specifically, the

548 prefrontal cortex and anterior striatum were shown to exhibit task-related activity modulation

549 (i.e., synchronization) under the former but not the latter condition (i.e., desynchronization). In

550 other words, neural networks for movement control were optimized, being regulated by smaller

551 scale neural circuits. This view is in line with the overall cortical synchronization on the task

552 observed on Type 1 and Type 4 performances and with the desynchronization observed on Type

5532 and Type 3 performances. In particular, the higher ERD during Type 3 performance is evident

554 in Figure 2 (Theta ERD/ERS). On the other hand, theta ERS reflected the activation of neural

555 networks involved in allocation of attention related to target stimuli rather than working memory

556 (Missonier et al., 2006). Finally, the ERS activity of Type 4 performance can be explained with

557 the lower level of control of the action exerted by the participants during this type of suboptimal

558 performance (see Table 1).

559 Moreover, we can hypothesize that the ERD/ERS pattern found in Type 1 performance is

560 likely related to a "default mode network" functioning, proper to autonomous skills and goal-

561 relevant attentional focus when approaching shot release (Doppelmeyr et al., 2008), similar to

562 what was found in golfers and basketball free throwers (Chuang, Huang \& Hung, 2013; Kao,

563 Huang, \& Hung, 2013). Excessive reinvestment in controlled processing undermines 
564 automaticity, and is related to higher cortical activity in the right parietal and frontal areas

565 (Broyd et al., 2009), which is evident during Type 3 performance. Gentili and colleagues (2011)

566 showed that with practice humans have the capacity to adapt their movements when challenged

567 with novel demands or task requirements. They found a cortical adaptation of frontal temporal

568 and parietal regions and a progressive idling of cortical rhythms in a learning paradigm of a

569 visuo-motor task (Gentili, Bradberry, Oh, Hatfield, \& Contreras Vidal, 2011). They suggested

570 that the update of motor plans in response to incoming sensory information could be mirrored in

571 theta band oscillation. There is also growing evidence that theta oscillations are linked to error

572 monitoring (Cavanagh, Cohen, \& Allen, 2009; Gevins \& Smith, 2000; Luu, Tucker, \& Makeig,

573 2004; Trujillo \& Allen, 2007; Yordanova et al., 2004). Error monitoring could explain the

574 difference we obtained in the maps for optimal (Type 1 and 2) and suboptimal (Type 3 and 4)

575 performance.

$576 \quad$ Findings in Alpha ERD/ERS (Figures 3 and 4) concur with the evidence indicating

577 relationships between: (1) low Alpha power and general cortical arousal, and (2) high Alpha

578 power and task-relevant attentional processing (Babiloni, 2008; Hatfield, 2013; Pfurtscheller \&

579 Lopes da Silva, 1999). Indeed, we observed a significant difference among the different types of

580 performance, particularly in FC2 site (see supplemental materials), with ERD patterns $(+1.12 \%)$

581 in low Alpha band for Type 3 performance. Thus, higher levels of general cortical arousal were

582 associated with suboptimal performance states. In Type 2 performance cortical activity was

583 synchronized on the task (-2.78\%). ERS was more evident in Type $4(-6.18 \%)$ and Type 1

584 performances (-9.14), indicating a low level of arousal and a minimal conscious control typical

585 of flow-like behaviour (Bortoli et al., 2012; Jackson \& Csikszentmihalyi, 1999). FC2 is one of

586 the electrodes that represent the area where attentive processes and control on core components 
587 of action can be integrated for the control of goal-directed and stimulus-driven attention

588 processes (Corbetta \& Schulman, 2012).

589 Furthermore, the increase in the neuronal predictive power for action that was observed in

590 the Fp1, F8, and P3 for high alpha band indicates that the optimization of neural networks can

591 occur qualitatively in the form of enhanced proficiency of the neuronal representation of action.

592 It is related to the visuo-spatial network that involves the switches between the performance

593 types reflected in the activity of fronto-parietal areas during a few seconds preceding the shot

594 release (i.e., Fpz, Fz, F8, Cp1; Matsuzaka, Akiyama, \& Mushiake, 2013). Moreover, the

595 involvement of the de-synchronization of the contralateral sensory-motor area (i.e., C3) is an

596 expression of the control of the right finger/hand (Pfurtscheller \& da Silva, 1999). The results are

597 also in agreement with the findings by Sauseng and colleagues (2005), who suggested that a shift

598 of attention selectively modulates excitability of the contralateral posterior parietal cortex and

599 that this posterior modulation of alpha activity is controlled by prefrontal regions.

600 In summary, the findings of the present study provided support to the construct of neural

601 proficiency in sport performance that underlies Type 1 and Type 2 performance states as

602 conceptualized in the MAP model (Bortoli et al., 2012), as well as in dual-process theories

603 (Furley et al., 2015) and ACT (see Wilson, 2008). According to this view, neural proficiency is

604 associated with Type 1 and Type 2 performance states, and mirrors the behaviour of elite athletes

605 when they use efficient or effortful processing to deal with task demands. This is in agreement

606 with the literature reviewed by Klimesh (2012), who highlighted how alpha ERS and ERD

607 reflect inhibition and release from inhibition, respectively. On the other hand, theta ERS reflects

608 top-down processes related to working memory and attention (Doppelmayr et al., 2008). 
From a neurophysiological perspective, successful goal-directed actions require the

610 individuals to flexibly adapt their behaviour to deal with performance problems or environment

611 changes, beyond correct action selection, planning, and execution (Ullsperger, Danielmeier, \&

612 Jocham, 2014). Performance optimization is ensured by a continuous monitoring of a wide

613 network of brain areas (such as the posterior medial frontal cortex), which detects and evaluates

614 deviations of actual from predicted states.

615 In conclusion, our findings provide evidence of distinct cortical processes underlying the

616 four types of performance states derived from the interaction between performance and attention

617 control levels. However, our methodological approach did not enable us to distinguish specific

618 cortical networks among the four types of performance. Further investigation using the tools of

619 graph theory (Bullmore \& Sporns, 2009) and dynamic functional connectivity (Hutchison et al.,

620 2013) within the framework of the MAP model, could provide a better understanding of the

621 functional connectivity and interactions among default mode, dorsal and ventral attention

622 network, sensory motor network, and visual network, typically involved in shooting

623 performance. The investigation of ERD/ERS patterns after shot release could provide important

624 information for a better understanding of the dynamics of brain oscillations during recovery from

625 a highly demanding task execution.

626 From an applied point of view, our findings can address neurofeedback training to

627 enhance performance in closed skill sports. Our findings can also help in choosing the proper

628 areas to apply transcranial electrical stimulation (Paulus, 2013) in order to facilitate cortical

629 plasticity, influencing the membrane potential of neurons and modulating spontaneous firing

630 rates. Indeed, during sport performance, the brain must dynamically integrate, coordinate, and

631 respond to internal and external stimuli across multiple time scales. 


\section{References}

634

635

636

637

638

639

640

641

642

643

644

645

646

647

648

649

650

651

652

653

654

Arrighi P, Bonfiglio L, Minichilli F, Cantore N, Carboncini MC, Piccotti E, et al. 2016. EEG theta dynamics within frontal and parietal cortices for error processing during reaching movements in a prism adaptation study altering visuo-motor predictive planning. PLoS ONE 11(3): e0150265. doi:10.1371/journal.pone.0150265

Babiloni C, Infarinato F, Marzano N, Iacoboni, M, Dassù F, Soricelli A, Rossini PM, Limatola C, Del Percio C. 2011. Intra-hemispheric functional coupling of alpha rhythms is related to golfer's performance: a coherence EEG study. International Journal of Psychophysiology 82:260-8.

Babiloni C, Marzano N, Iacoboni M, Infarinato F, Aschieri P, Buffo P, .... \& Del Percio C. 2010. Resting state cortical rhythms in athletes: a high-resolution EEG study. Brain Research Bullettin 81:149-56.

Babiloni C, Del Percio C, Iacoboni M, Infarinato F, Lizio R, Marzano N, . . Eusebi F. 2008. Golf putt outcomes are predicted by sensorimotor cerebral EEG rhythms. Journal of Physiology 586:131-139. doi:10.1113/jphysiol.2007.141630

Beilock SL, \& Carr TH. 2001. On the fragility of skilled performance: What governs choking under pressure? Journal of Experimental Psychology: General 130:701-725. doi:10.1037/0096-3445.130.4.701

Bertollo M, Bortoli L, Gramaccioni G, Hanin Y, Comani S, Robazza C. 2013. Behavioural and psychophysiological correlates of athletic performance: A test of the multi-action plan model. Applied Psychophysiology Biofeedback 38:91-99. doi: 10.1007/s10484-013-9211- 
655 Bertollo M, di Fronso S, Filho E, Lamberti V, Ripari P, Reis VM, Comani S, Bortoli L, Robazza

656 C. 2015 . To focus or not to focus: Is attention on the core components of action beneficial 657 for cycling performance? Sport Psychologist 29:110-119. Doi: 10.1123/tsp.2014-0046

658 Bishop SJ. 2009. Trait anxiety and impoverished prefrontal control of attention. Nature $659 \quad$ Neuroscience 12:92-98. doi: 10.1038/nn.2242

660 Bortoli L, Bertollo M, Hanin Y, Robazza C. 2012. Striving for excellence: A multi-action plan 661 intervention model for shooters. Psychology of Sport and Exercise 13:693-701.

662

663

664

665

666

667

668

669

670

671

672

673

674

675

Broyd SJ, Demanuele C, Debener S, Helps SK, James CJ, \& Sonuga-Barke EJS. 2009. Defaultmode brain dysfunction in mental disorders: A systematic review. Neuroscience and Biobehavioral Reviews 33:279-296. doi:10.1016/j.neubiorev.2008.09.002

Bullmore E \& Sporns O. 2009. Complex brain networks: Graph theoretical analysis of structural and functional systems. Nature Reviews Neuroscience 10:186-198. doi:10.1038/nrn2575

Callan DE \& Naito E. 2014. Neural processes distinguishing elite from expert and novice athletes. Cognitive Behavioural Neurology 27:183-8. doi:10.1097/WNN.0000000000000043.

Carson HJ \& Collins D. 2016. The fourth dimension: A motoric perspective on the anxietyperformance relationship. International Review of Sport and Exercise Psychology 9:1-21. doi:10.1080/1750984X.2015.1072231

Cavanagh JF, Cohen MX, \& Allen JJ. 2009. Prelude to and resolution of an error: EEG phase synchrony reveals cognitive control dynamics during action monitoring. The Journal of Neuroscience 29:98-105. doi:10.1523/JNEUROSCI.4137-08.2009 
676 Cheng M, Hung C, Huang C, Chang Y, Lo L, Shen C, \& Hung T. 2015a. Expert-novice

677

678

679

680

681

682

683

684

685

686

687

688

689

690

691

692

693

694

695

696

697

698

differences in SMR activity during dart throwing. Biological Psychology, 110: 212-218. doi:10.1016/j.biopsycho.2015.08.003

Cheng MY, Huang CJ, Chang YK, Koester D, Schack T, Hung TM. 2015b. Sensorimotor rhythm neurofeedback enhances golf putting performance. Journal of Sport and Exercise Psychology. 37:626-36. doi: 10.1123/jsep.2015-0166

Chuang LY, Huang CJ, \& Hung TM. 2013. The differences in frontal midline theta power between successful and unsuccessful basketball free throws of elite basketball players. International Journal of Psychophysiology 90:321-328. doi:10.1016/j.ijpsycho.2013.10.002

Comani S, Bortoli L, Di Fronso S, Fiho E, De Marchis C, Schmid M, . . Bertollo M. 2014. ERD/ERS patterns of shooting performance within the multi-action plan model. IFMBE Proceedings 41:141-144. doi:10.1007/978-3-319-00846-2_35

Cooke A, Kavussanu M, Gallicchio G, Willoughby A, Mcintyre D, \& Ring C. 2014. Preparation for action: Psychophysiological activity preceding a motor skill as a function of expertise performance outcome and psychological pressure. Psychophysiology 51:374-384. doi:10.1111/psyp.12182

Cooke A. 2013. Readying the head and steadying the heart: A review of cortical and cardiac studies of preparation for action in sport. International Review of Sport and Exercise Psychology 6:122-138. doi:10.1080/1750984X.2012.724438

Coombes SA, Higgins T, Gamble KM, Cauraugh JH, \& Janelle C.M. 2009. Attentional Control Theory: Anxiety Emotion and Motor Planning. Journal of Anxiety Disorders 23:10721079. 
699 Corbetta M. \& Shulman GL. 2002. Control of goal-directed and stimulus-driven attention in the $700 \quad$ brain. Nature Reviews Neuroscience 3:201-215.

701

702

703

704

705

706

707

708

709

710

711

712

713

714

715

716

717

718

719

720

Del Percio C, Infarinato F, Marzano N, Iacoboni M. Aschieri P, Lizio R, Soricelli A, Limatola C, Rossini PM, Babiloni C. 2011. Reactivity of alpha rhythms to eyes opening is lower in athletes than non-athletes: a high-resolution EEG study. International Journal of Psychophysiology 82:240-7.

Del Percio C, Babiloni C, Bertollo M, Marzano N, Iacoboni M, Infarinato F, ... Eusebi F. 2009. Visuo-attentional and sensorimotor alpha rhythms are related to visuo-motor performance in athletes. Human Brain Mapping 30:3527-3540. doi:10.1002/hbm.20776

Del Percio C, Babiloni C, Marzano N, Iacoboni M, Infarinato F, Vecchio F, . . . Eusebi F. 2009. "Neural efficiency" of athletes' brain for upright standing: A high-resolution EEG study. Brain Research Bulletin 79:193-200. doi:10.1016/j.brainresbull.2009.02.001

Del Percio C, Rossini PM, Marzano N, Iacoboni M, Infarinato F, Aschieri P, . . Eusebi F. 2008. Is there a "neural efficiency" in athletes? A high-resolution EEG study. NeuroImage 42:1544-1553. doi:10.1016/j.neuroimage.2008.05.061

Del Percio C, Infarinato F, Iacoboni M, Marzano N, Soricelli A, Aschieri P, . . Babiloni C. 2010. Movement-related desynchronization of alpha rhythms is lower in athletes than non-athletes: A high-resolution EEG study. Clinical Neurophysiology 121:482-491. doi:10.1016/j.clinph.2009.12.004

di Fronso S. Robazza C, Filho E, Bortoli L, Comani S, \& Bertollo M. 2016. Neural markers of performance states in an Olympic Athlete: An EEG case study in air-pistol shooting. Journal of Sports Science \& Medicine 15:215-223. 
721 Doppelmayr M, Finkenzeller T, \& Sauseng P. 2008. Frontal midline theta in the pre-shot phase

722

723

724

725

726

727

728

729

730

731

732

733

734

735

736

737

738

739

740

741

742

743

of rifle shooting: Differences between experts and novices. Neuropsychologia 46:14631467. doi:10.1016/j.neuropsychologia.2007.12.026

Ericsson KA. 2007. Deliberate practice and the modifiability of body and mind: Toward a science of the structure and acquisition of expert and elite performance. International Journal of Sport Psychology 38:4-34.

Eysenck MW, Derakshan N. 2011. New perspectives in attentional control theory. Personality and Individual Differences 50:955-960. DOI: 10.1016/j.paid.2010.08.019

Eysenck MW \& Calvo MG. 1992. Anxiety and performance: The processing efficiency theory. Cognition and Emotion 6:409-434.

Eysenck MW, Derakshan N, Santos R, \& Calvo MG. 2007. Anxiety and cognitive performance: Attentional control theory. Emotion 7:336-353.

Filho E, di Fronso S, Mazzoni C, Robazza C, Bortoli L, \& Bertollo M. 2015. My heart is racing! Psychophysiological dynamics of skilled racecar drivers. Journal of Sports Sciences 33:945-959. doi:10.1080/02640414.2014.977940

Furley P, Schweizer G, \& Bertrams A. 2015. The two modes of an athlete: Dual-process theories in the field of sport. International Review of Sport and Exercise Psychology 8:106-124. doi:10.1080/1750984X.2015.1022203

Gentili RJ, Bradberry TJ, Oh H, Hatfield BD, \& Contreras Vidal JL. 2011. Cerebral cortical dynamics during visuomotor transformation: Adaptation to a cognitive-motor executive challenge. Psychophysiology 48:813-824. doi:10.1111/j.1469-8986.2010.01143.x

Gevins A, \& Smith ME. 2000. Neurophysiological measures of working memory and individual differences in cognitive ability and cognitive style. Cerebral Cortex 10:829-839. 
744 Haier RJ, Jung RE, Yeo RA, Head K, Alkire MT. 2004. Structural brain variation and general

745

746

747

748

749

750

751

752

753

754

755

756

757

758

759

760

761

762

763

764

765 intelligence. Neuroimage. 23:425-433.

Haier RJ, Siegel BVJr, MacLachlan A, Soderling E, Lottenberg S, Buchsbaum MS. 1992).

Regional glucose metabolic changes after learning a complex visuospatial/motor task: a positron emission tomographic study. Brain Research 570:134-143.

Haier RJ, Siegel BV, Nuechterlein KH, Hazlett E, Wu JC, Paek J, Browning HL, Buchsbaum MS. 1988. Cortical glucose metabolic rate correlates of abstract reasoning and attention studied with positron emission tomography. Intelligence 12:199-217.

Hanin YL. 2007. Emotions in sport: Current issues and perspectives. In G. Tenenbaum \& R. Eklund (Eds.) Handbook of sport psychology (3rd ed. pp. 31-58). Hoboken: Wiley.

Hatfield BD, Costanzo ME, Goodman RN, Lo C, Oh H, Rietschel JC. . . Haufler A. 2013. The influence of social evaluation on cerebral cortical activity and motor performance: A study of "real-life" competition. International Journal of Psychophysiology 90:240-249. doi:10.1016/j.ijpsycho.2013.08.002

Hatfield BD \& Kerick SE. 2007.The psychology of superior sport performance: a cognitive and affective neuroscience perspective. In Eklund RC \& Tenenbaum G editors. Handbook of sport psychology 3rd ed. John Wiley \& Sons Inc; pp. 84-109.

Hill DM, Hanton S, Matthews N, \& Fleming S. 2010. Choking in sport: A review International Review of Sport and Exercise Psychology 3:24-39. doi: 10.1080/17509840903301199

Hutchison RM, Womelsdorf T, Allen EA, Bandettini PA, Calhoun VD, Corbetta M, ... Chang C. 2013. Dynamic functional connectivity: Promise issues and interpretations. Neuroimage 80:360-378. doi:10.1016/j.neuroimage.2013.05.079 
766 Huynh H, Feldt LS. 1976. Estimation of the Box correction for degrees of freedom from sample

767 data in randomized block and split-plot designs. Journal of Education \& Behavioral

$768 \quad$ Statisitcs 1:69-82.

769 Jackson SA, \& Csikszentmihalyi M. 1999. Flow in sports: The keys to optimal experiences and 770 performances. Champaign IL: Human Kinetics.

771 Kao SC, Huang CJ, \& Hung TM. 2013. Frontal midline theta is a specific indicator of optimal attentional engagement during skilled putting performance. Journal of Sport and Exercise Psychology 35:470-478.

774

775

776

777

778

779

780

781

782

783

784

785

786

787

788

Klimesch W. 1999. EEG alpha and theta oscillations reflect cognitive and memory performance: a review and analysis. Brain Research Review 29:169-95.

Klimesch W. 2012. Alpha-band oscillations, attention and controlled access to stored information. Trends in Cognitive Sciences 16:606-617. doi:10.1016/j.tics.2012.10.007

Luu P, Tucker DM, Makeig S. 2004. Frontal midline theta and the error-related negativity: neurophysiological mechanisms of action regulation. Clinical Neurophysiology $115: 1821-1835$.

Magill R \& Anderson D. 2014. Motor Learning and Control: Concepts and Applications (10 ${ }^{\text {th }}$ edition) McGraw-Hill pp 512

Masters R, \& Maxwell J. 2008. The theory of reinvestment. International Review of Sport and Exercise Psychology 1:160-183. doi:10.1080/17509840802287218

Matsuzaka Y, Akiyama T, \& Mushiake H. 2013. Neuronal representation of task performance in the medial frontal cortex undergoes dynamic alterations dependent upon the demand for volitional control of action. Experimental Brain Research 229:395-405.

doi:10.1007/s00221-013-3454-z 
789 Maxwell JP, Masters RS \& Eves F. 2000. From novice to no know-how: A longitudinal study of 790 implicit motor learning. Journal of Sports Sciences 18:111-120.

791 Missonnier P, Deiber MP, Gold G, Millet P, Gex-Fabry Pun M, Fazio-Costa L, . . Ibáñez V. 792 2006. Frontal theta event-related synchronization: Comparison of directed attention and 793 794 795 Brain Stimulation 6(2):122-129. doi:10.1016/j.brs.2012.03.001.

798

799

800

801

802

803

804

805

806

807

808 809

810

811 working memory load effects. Journal of Neural Transmission 113:1477-1486. doi:10.1007/s00702-005-0443-9

Morgan HM, Jackson MC, van Koningsbruggen MG, Shapiro KL, Linden DEJ. 2013. Frontal and parietal theta burst TMS impairs working memory for visual-spatial conjunctions.

Nussbaumer D, Grabner RH, \& Stern E. 2015. Neural efficiency in working memory tasks: The impact of task demand. Intelligence 50:196-208. doi:10.1016/j.intell.2015.04.004

Oudejans RRD, Kuijpers W, Kooijman CC, \& Bakker FC. 2011. Thoughts and attention of athletes under pressure: Skill-focus or performance worries? Anxiety Stress and Coping 24:59-73. doi:10.1080/10615806.2010.481331

Park JL, Fairweather MM, \& Donaldson DI. 2015. Making the case for mobile cognition: EEG and sports performance. Neuroscience and Biobehavioral Reviews 52:117-130. doi:10.1016/j.neubiorev.2015.02.014

Paulus W. 2011: Transcranial electrical stimulation (tES- tDCS; tRNS tACS) methods. Neuropsychological Rehabilitation: An International Journal 21:602-617.

Pfurtscheller G \& Lopes da Silva FH. 1999. Event-related EEG/MEG synchronization and desynchronization: Basic principles. Clinical Neurophysiology 110:1842-1857.

Poldrack RA. 2015. Is "efficiency" a useful concept in cognitive neuroscience? Developmental Cognitive Neuroscience 11:12-17. doi:10.1016/j.dcn.2014.06.001 
812 Righi S, Mecacci L, \& Viggiano MP. 2009. Anxiety cognitive self-evaluation and performance:

813

814

815

816

817

818

819

820

821

822

823

824

825

826

827

828

829

830

831

832

833

ERP correlates Journal of Anxiety Disorders 23:1132-1138. doi:

10.1016/j.janxdis.2009.07.018

Robazza C, Bertollo M, Filho E, Hanin Y, \& Bortoli L. 2016. Perceived control and hedonic tone fluctuations during performance in elite shooters. Research Quarterly for Exercise and Sport. Submitted

Sauseng P, Klimesch W, Stadler W, Schabus M, Doppelmayr M, Hanslmayr S, ... Birbaumer N. 2005. A shift of visual spatial attention is selectively associated with human EEG alpha activity. European Journal of Neuroscience 22:2917-2926. doi:10.1111/j.14609568.2005.04482.x

Savostyanov AN, Tsai AC, Liou M, Levin EA, Lee JD, Yurganov AV, \& Knyazev GG. 2009. EEG-correlates of trait anxiety in the stop-signal paradigm Neuroscience Letters 449:112116. doi: 10.1016/j.neulet.2008.10.084

Swann C, Keegan R, Crust L, \& Piggott D. 2016. Psychological states underlying excellent performance in professional golfers: "Letting it happen" vs. "making it happen". Psychology of Sport and Exercise 23:101-113. doi: 10.1016/j.psychsport.2015.10.008

Trujillo LT, \& Allen JJ. 2007. Theta EEG dynamics of the error-related negativity. Clinical. Neurophysiology 118:645-668.

Ullsperger M, Danielmeier C, \& Jocham G. 2014. Neurophysiology of performance monitoring and adaptive behavior. Physiological Reviews 94:35-79. doi:10.1152/physrev.00041.2012

Vecchio F, Del Percio C, \& Babiloni C. 2012. Is there a neural efficiency in the athlete's brain? International Journal of Psychophysiology 85:296-297. 
834 Wilson M. 2008. From processing efficiency to attentional control: A mechanistic account of the 835 anxiety-performance relationship. International Review of Sport and Exercise Psychology

836 1:184-201. doi: 10.1080/17509840802400787

837 Wilson MR, Wood G, \& Vine SJ. 2009. Anxiety, attentional control and performance

838 impairment in penalty kicks. Journal of Sport \& Exercise Psychology 31:761-775.

Yordanova J, Falkenstein M, Hohnsbein J, Kolev V. 2004. Parallel systems of error processing 840 in the brain. Neuroimage 22:590-602.

841 Zanow F \& Knosche TR. 2004. ASA-Advanced source analysis of continuous and event-related EEG/MEG signals. Brain Topography 16:287-290. 